\title{
Pulmonary Tuberculosis Diagnosis Using Artificial Neural Networks
}

Karam Hatim Albajary

karamhatim@uomosul.edu.iq
Mohammed Abd-alraheem Hamdi

mohammed@uomosul.edu.iq

Computer science and Mathematics College

University of Mosul, Iraq

\section{Received on: 07/10/2008}

\section{ABSTRACT}

Accepted on: 04/12/2008

This research can detecting the Tuberculosis by using the artificial neural networks, the idea of this research is to design a system that receive the information of patient and give these information to Hamming and Maxnet network which doing the comparison between these information with constant values of human body that stored in network. The system is implemented about many humans (infected and not infected) which has been entered their information to the data base of system, and programmed the system by using visual basic 6.0 with Microsoft access software to build the data base.

Keywords: neural networks, visual basic data base, Diagnosis diseases.

$$
\begin{aligned}
& \text { تثخيص مرض التدرن الرئوي (السل) باستخدام الثبكات العصبية الاصطناعية }
\end{aligned}
$$

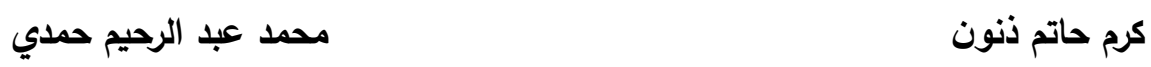

$$
\begin{aligned}
& \text { كلية علوم الحاسبات واليراضيات، جامعة الموصل }
\end{aligned}
$$

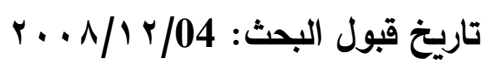

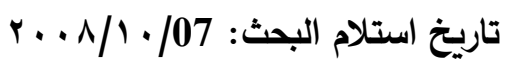

$$
\begin{aligned}
& \text { الملخص } \\
& \text { تم في هذا البحث تمييز مرض التدرن الرئوي أو ما يعرف بالسل عن طريق استخدام الثبكات العصبية }
\end{aligned}
$$

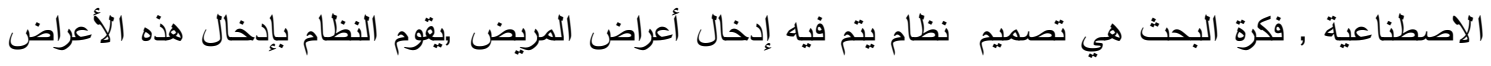

$$
\begin{aligned}
& \text { إلى الثبكة العصبية "hamming and maxnet" التي تقوم بالتمييز. لقد تم اختبار النظام على عدد من } \\
& \text { الأشخاص المصابين وغير المصابين والذين تم إدخال المعلومات منهم الى قاعدة البيانات التابعة للنظام المبرمج } \\
& \text { باستخدام لغة } 6.0 \text { visual basic مع قاعدة البيانات باستخدام Microsoft Access وأعطى النظام إمكانية } \\
& \text { تمييز عالية جدا. } \\
& \text { الكلمات المفتاحية: الثبكات العصبية، قواعد بيانات visual basic، تشخيص الامراض. } \\
& \text { 1. مقدمة }
\end{aligned}
$$


العلامة المرضية من خلال إدخالها وبعدها ستكون قادرة على إيجاد العينة المخزنة الكاملة والتي تمثل أفضل

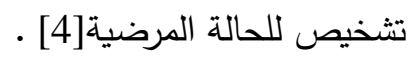
2. 2 أسباب انتشار المرض يعتبر مرض التدرن الرئوي من المشاكل التي تعانيها كثير من البلدان ومن أهم الأسباب التي يعزى إليها انتشار هذا المرض بين أهالي أي بلد هي كالآتي [7]:

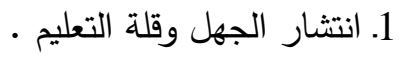

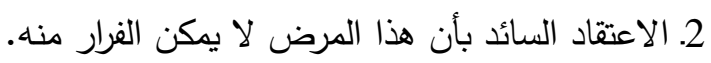
3 ـ عدم توفر الوعي الصحي والجهل بالمستويات الصحية. 4. عدم توفر عوامل نشر التثقيف الصحي بين المواطنين. 5. نزوح كثير من أهل البادية الذين لا تتوفر لديهم المناعة ضد المرض إلى المدن حيث تتوفر الكثير من عوامل انتشار المرض.

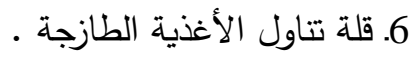
7. طريقة تتاول الغذاء والشراب التي تتنافى ومبادئ الصحة العامة والسائدة بين بعض المواطنين.

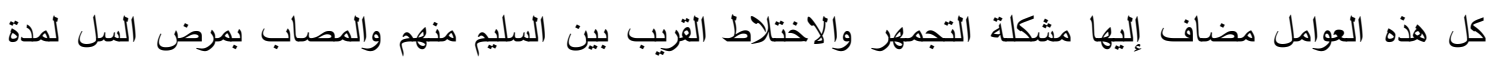
طويلة تحت ظروف صحية عامة وغذائية سيئة للغاية، كل هذه العوامل أسباب قوية تؤدي إلى كثرة انتشار المرض.

3. الشبكات العصبية الاصطناعية Artificial Neural Networks (ANN) هي تقنيات حسابية مصممة لمحاكاة الطريقة التي يؤدي بها الدماغ البشري مهمة معينة وذلك عن طريق معالجة ضخمة موزعة على التوازي ومكونة من وحدات معالجة بسيطة، هذه الوحدات ما هي إلا عناصر حسابية تسمى عصبونات أو عقد ( Nodes, Neurons ) والتي لها خاصية عصبية من حيث أنها تقوم بتخزين المعرفة العملية والمعلومات التجريبية لتجعلها متاحة للمستخدم وذلك عن طريق ضبط الأوزان. يكمن احد أسباب تفوق الدماغ البشري في قدرته على معالجة المعطيات بأكثر من مجموعة من الخلايا العصبية

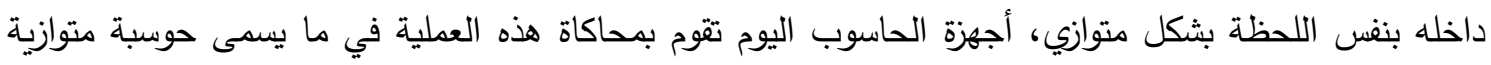

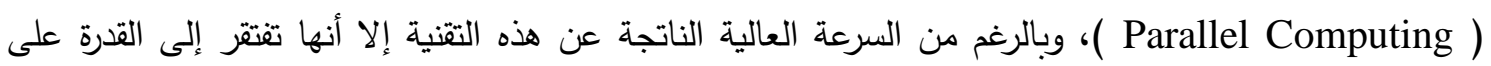
الاستقلال بحل المشكلة، بمعنى أن النظام غير قادر على حل المشكلة باستعمال المعطيات المدخلة لوحدها بدون

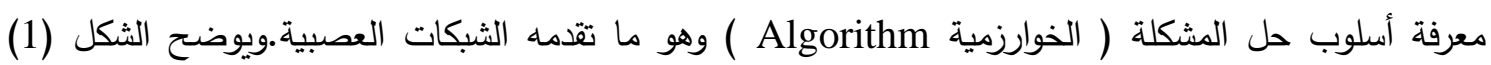

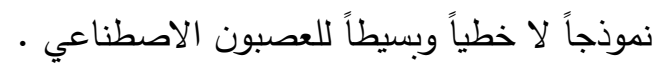

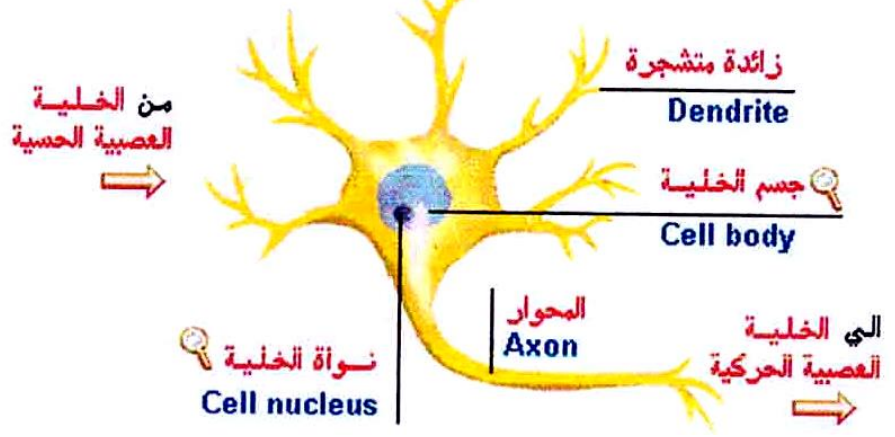




$$
\text { الثكل (1) نموذجاً لا خطياً وبسيطاً للعصبون الاصطناعي }
$$

إذ أن الـ ANN تتشابه مع الدماغ البشري في أنها تكتسب المعرفة بالتدريب وتخزن هذه المعرفة باستخدام قوى وصل داخل العصبونات تسمى الأوزان التشابكية، وهناك أيضا تثابه عصبي حيوي مما يعطي الفرصة لعلماء البايولوجيا في الاعتماد على ANN لفهم تطور الظواهر الحيوية [1].

\section{4. مكونات الثبكة العصبونية الاصطناعية}

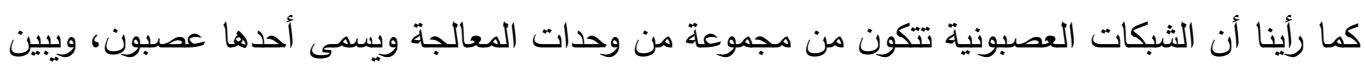

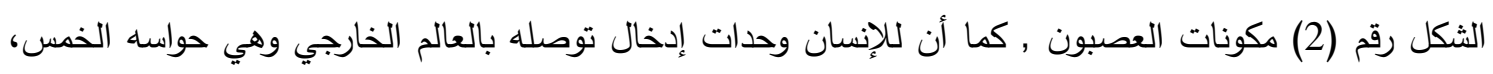
فكذلك الشبكات العصبية تحتاج لوحدات إدخال ووحدات معالجة يتم فيها عمليات حسابية تضبط بها الأوزان

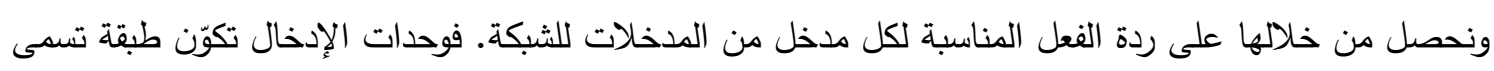
طبقة المدخلات ووحدات المعالجة تكوّن طبقة المعالجة وهي التي تخرج نواتج الثبكة. وبين كل طبقة من هذه من الهن الطبقات هناك طبقة من الوصلات البينية التي تربط كل طبقة بالطبقة التي تليها والتي يتم فيها ضبط الأوزان

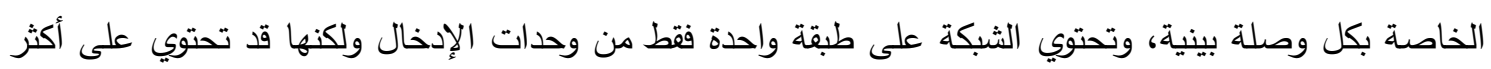
من طبقة من طبقات المعالجة [3].

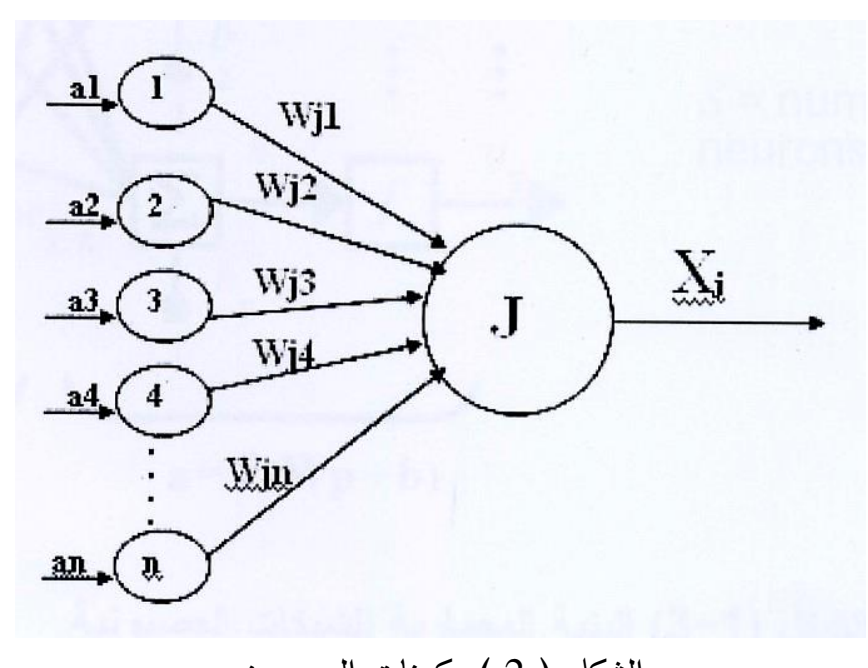

الثكل ( 2 - 2 2 مكونات العصبون

نلاحظ من الثكل ( 2 ) أن العصبون يتألف من :

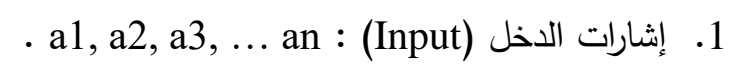

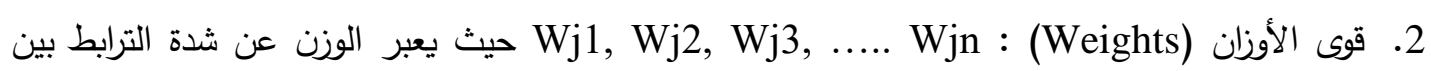
عنصر قبله وعنصر بعده.

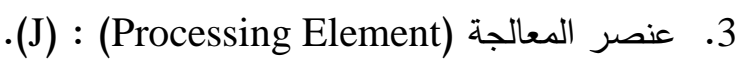

(Xj) : (Output) ( 
(Hamming \& Maxnet) شبكة

هي عبارة عن شبكة تصنيف تتألف من طبقتين (Two Layers) تستخدم لتصنيف المتجهات التي تكون مكوناتها من النوع ثنائي القطبية (Bipolar (-1,1)،ويبين الثكل التالي المخطط الصندوقي لهذه الثبكة .

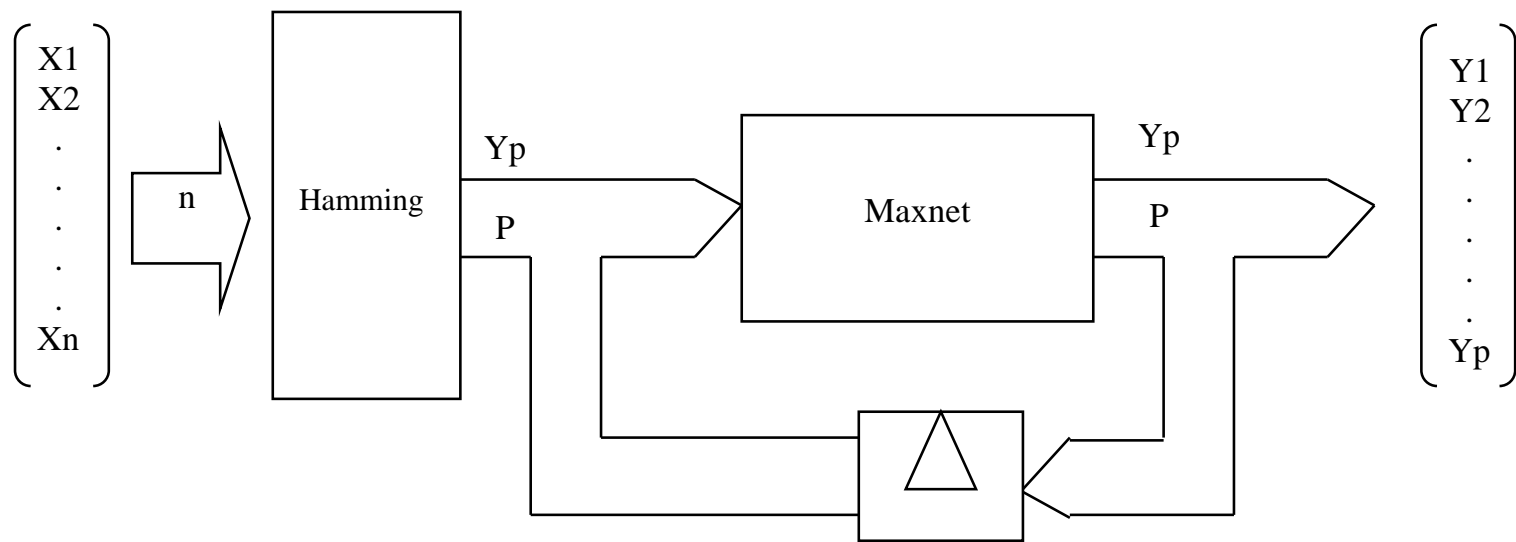

Hamming \& Maxnet الثكل ( 3 ) المخطط الصندوقي لثبكة

ويتضح من المخطط الصندوقي أن شبكة Hamming تمثل الطبقة الأولى (First Layer) من شبكة والتي يقع على عاتقها إجراء عملية التصنيف الأساسية إلى (pamming \& Maxnet ) تمتلك هذه الثبكة (p) من الإخراجات والاستجابة الأقوى للخلية أو العصب (Neuron) تشير إلى الخى الخلية التي يميلك اقل مقدار من مسافة Hamming أي (Minimum Hamming Distance) بين الإدخال والصنف الذي

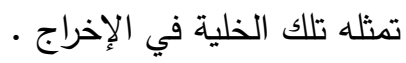
وتعتبر شبكة Hamming من الثبكات ذات التغذية الأمامية (feed Forward network) والتي تعتمد أسلوب المطابقة (Matching) بين الإدخال والنماذج المخزونة في الثبكة . أما الطبقة الثانية (Second Layer) من شبكة التصنيف هي شبكة (Maxnet) التي تعمل بشكل يعتمد على التغذية العكسية (Feedback) ،وهي أداة فقط لقمع العقد (Nodes) الخارجة من أول طبقة التي تحمل

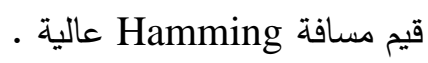
أي أن شبكة maxnet تبقي الخلية أو العقدة التي تمتلك أعلى قيمة في الإخراج واقل مسافة من شبكة Hamming من الطبقة الأولى [3]. ومن مميزات شبكة Hamming \& Maxnet

1. تمثل شبكة Maxnet و Hamming مصنّا مثاليا للنماذج بأقل خطأ ممكن أي هي Minimum Error ) .Bit Classifier) Optimum 2. تمتلك سرعة عالية في تمييز الأنماط العشوائية.

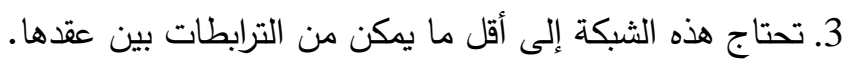

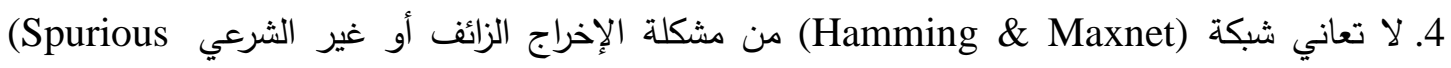

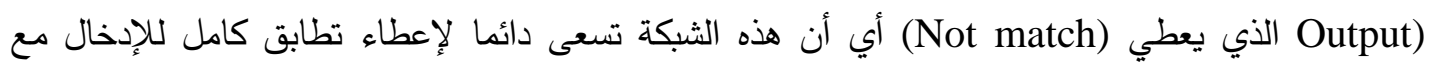
الأمثلة أو اقرب تطابق ممكن ولا تعطي أبدا (Not match). 
5. البساطة في التمثيل والتنفيذ والمتابعة.

[4] Hamming \& Maxnet 6

Step (1) : Specify the example $S$ ij.

Step (2) : Fixed the weight matrix for Hamming net.

$\mathrm{Wh}=1 / 2\left(\begin{array}{cc}\mathrm{S} 1^{1} & \mathrm{~S} 2^{1 \cdots} \mathrm{Sn}^{1} \\ \cdot & \cdot \\ \mathrm{S} 1^{\mathrm{m}} & \mathrm{S} 2^{\mathrm{m} \cdots} \mathrm{Sn}^{\mathrm{m}}\end{array}\right) \mathrm{m} * \mathrm{n}$

Where $\mathrm{n}=$ No. of input vector $\& \mathrm{~m}=$ No. of example

Step (3) : Find $\theta$ where $\theta=\mathrm{n} / 2$

$$
\theta=\left(\begin{array}{c}
\theta \\
\theta \\
\cdot \\
\cdot \\
\cdot
\end{array}\right) \quad \begin{aligned}
& \mathrm{j}=1 \ldots \mathrm{m} \\
& \mathrm{n}=\text { No. of input vector }
\end{aligned}
$$

Step (4) : Specify the testing input vector $X$

$X=\left(\begin{array}{c}X 0 \\ X 1 \\ \cdot \\ \cdot \\ \cdot \\ X n\end{array}\right) \quad \begin{aligned} & \mathrm{j}=1 \ldots \mathrm{m}=\text { No. of input vector } \\ & \end{aligned}$

Step (5) : Find the output form Hamming net

Net $\mathrm{j}=\mathrm{wh}^{*} \mathrm{X}+\theta$

Step (6) : Find $Y^{k}{ }_{j}=1 / \mathrm{n} *$ net $\mathrm{j}$

Step (7) : Specify the value of $\varepsilon$ when $0<\varepsilon<1 / \mathrm{m} \&$ Find Wm

$\mathrm{Wm}=\left(\begin{array}{ccc}1 & -\varepsilon \cdots-\varepsilon \\ -\varepsilon & 1 & -\varepsilon \\ -\varepsilon & -\varepsilon & 1\end{array}\right) \quad \mathrm{m} * \mathrm{~m}$ 
Step (8) : Find $Y^{k+1}$

$Y^{k+1}=\mathrm{r}\left(\mathrm{Wm} * Y^{k}\right)$ where $\mathrm{r}=\begin{gathered}0 \text { if net } \\ \text { net if net ?o }\end{gathered}$

Step (9) : Repeat Step (7) until convergence.

Hamming \& Maxnet 7

لو فرضنا أن لدينا ثلاث أنماط كل نمط من تسع قيم فإن البنية الهندية (المعمارية) للشبكة ستكون كلآتي : - n

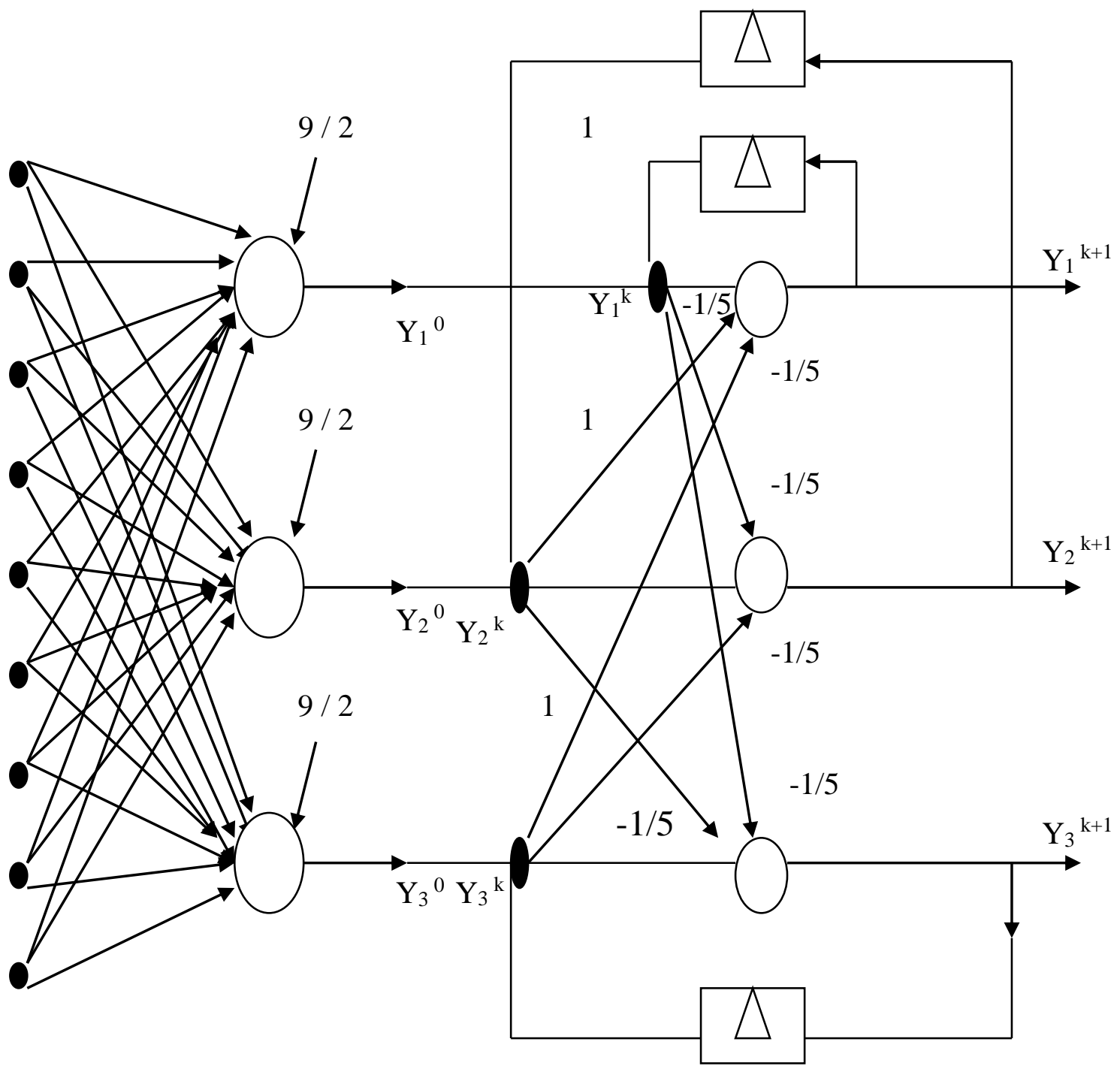

Hamming net Max net

الثكل ( 4 ( )

Hamming \& Maxnet معمارية 
8

لقد تم تطبيق الثبكة العصبية على المرض عن طريق الرمز إلى أعراض المريض بـ (1 أو 1-) وذلك لان شبكة Hamming \& maxnet تتعامل مع القيم بشكل Bipolar وتم خزن هذه القيم في متجه الإدخال

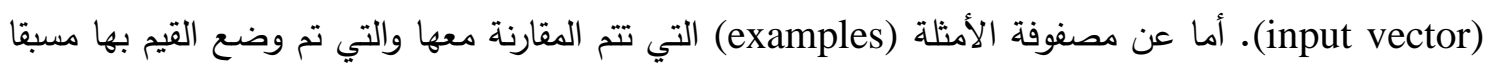

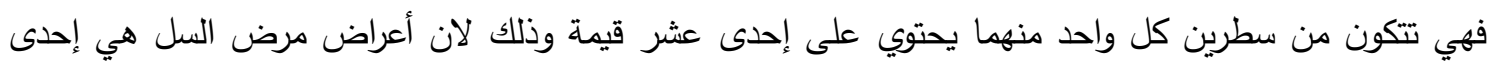

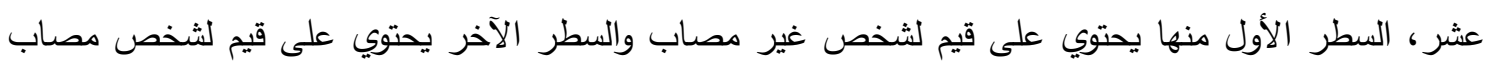

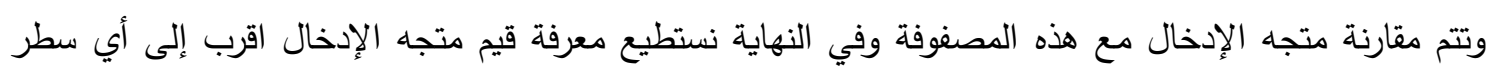

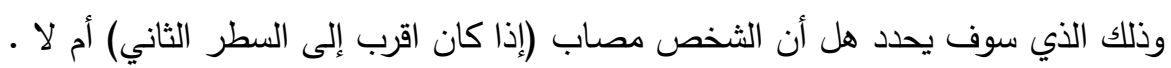

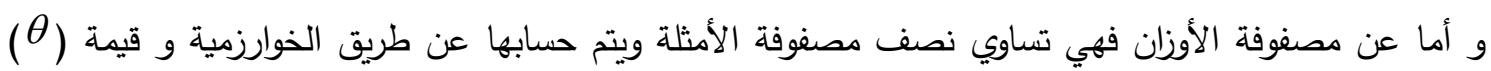

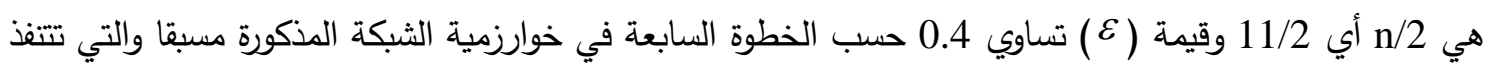
خطواتها واحدة بعد الأخرى إلى أن تتم الوصول إلى إلى التمييز . و سوف نوضح الآن الواجهات الرئيسية للبرنامج حيث يبين (الثكل (5)) واجهة التئل التمارة معلومات

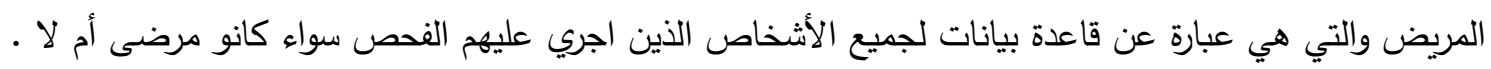

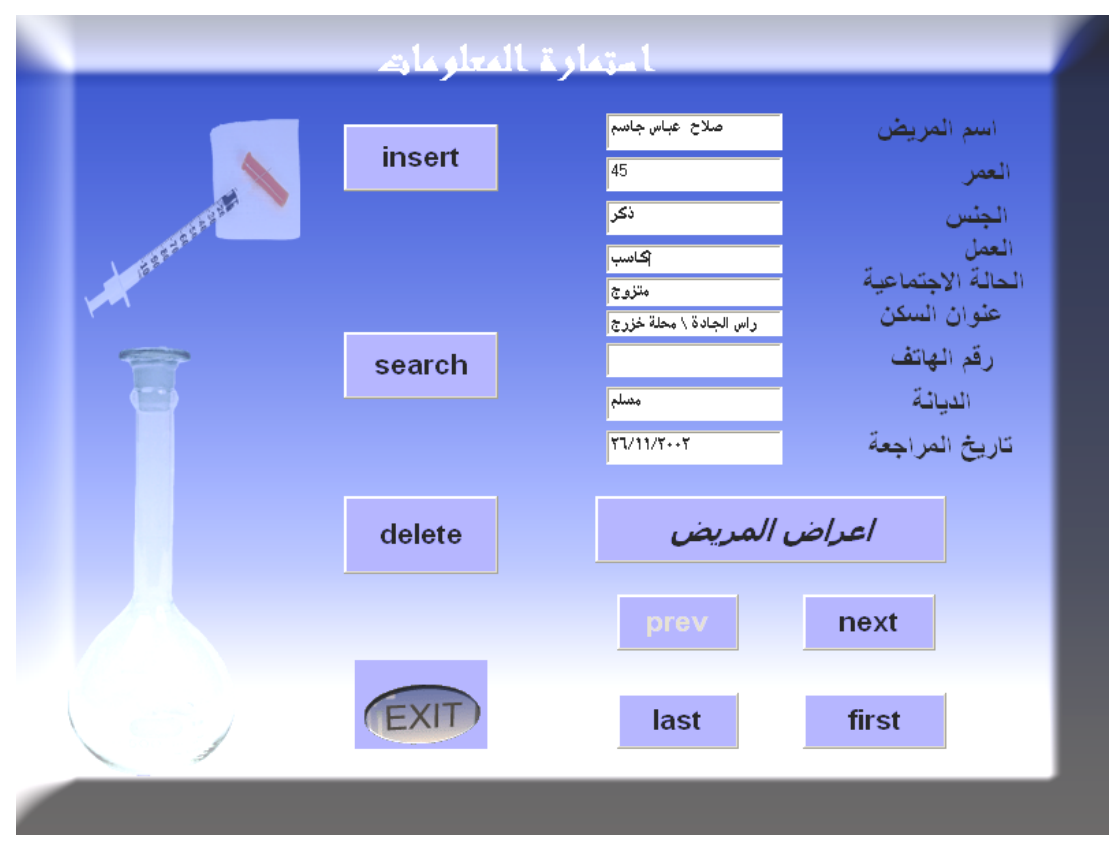

$$
\begin{aligned}
& \text { الثكل ( } 5 \text { ) واجهة استمارة المعلومات للمريض } \\
& \text { وللبحث القدرة على أداء العمليات الآتية : }
\end{aligned}
$$

: عملية الإضافة*

تشمل هذه العملية إمكانية إضافة شخص إلى قاعدة البيانات المكونة مسبقا ليتم فحصه لاحقا و عن طريق

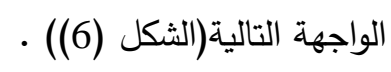




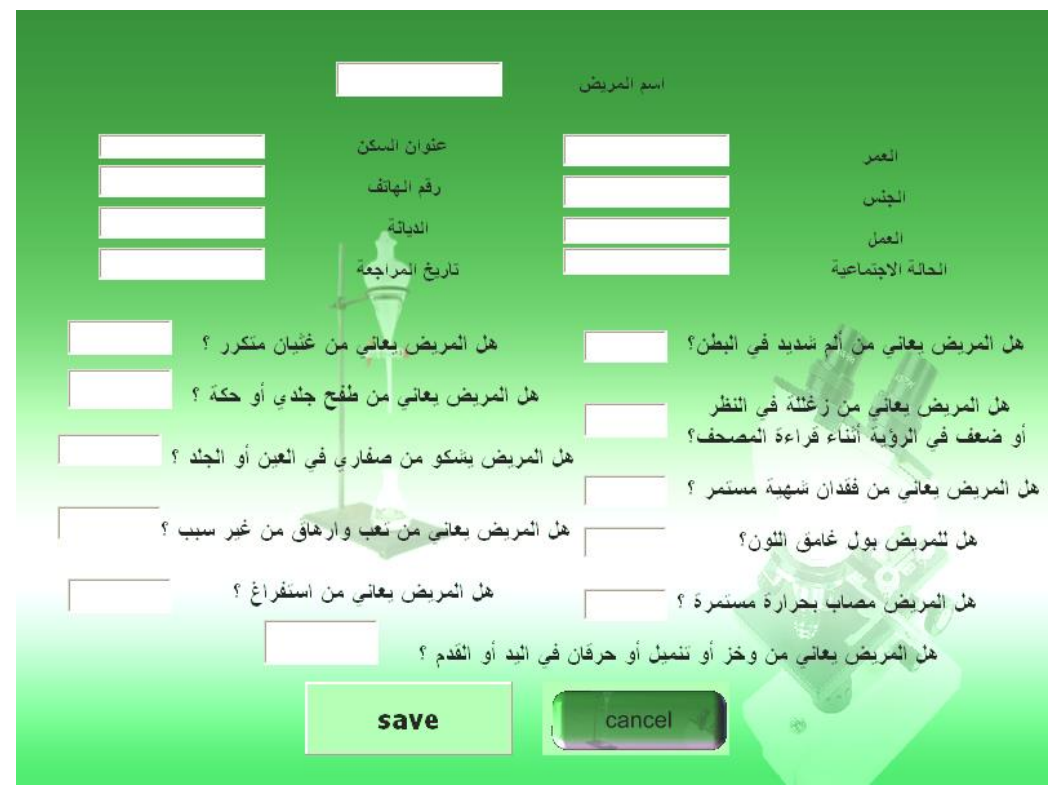

الشكل ( 6 ( ) واجهة خزن معلومات المريض

*عملية البحث :

وتتم في هذه العملية البحث عن أي شخص في قاعدة البيانات عن طريق كتابة اسمه ليتم إجراء الفحص عليه أو

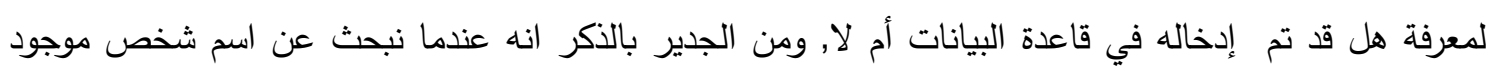
يظهر اسمه في استمارة المعلومات مباشرة وعندها يمكن إجراء الفحص عليه والتأكد من حالته بسهولة. والثكل التالي يوضح واجهة البحث.

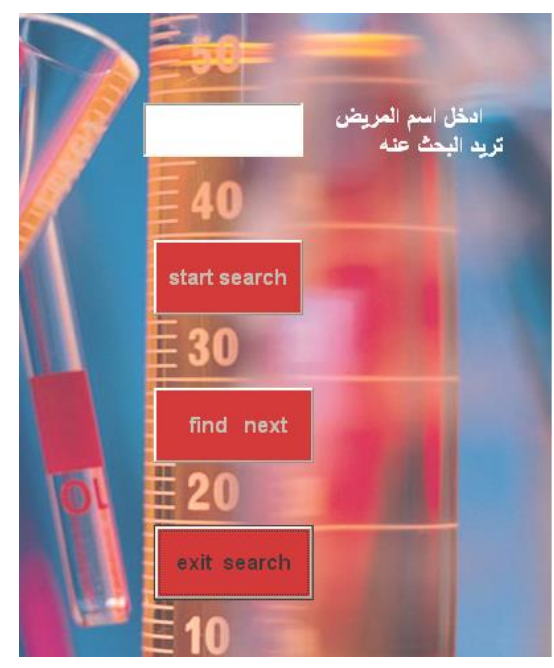

الثكل ( 7 ) واجهة البحث عن اسم المريض 
:عملية الحذف:

للبحث إمكانية حذف معلومات أي شخص من استمارة المعلومات والتي تمثل قاعدة البيانات نظرا لخروجه من

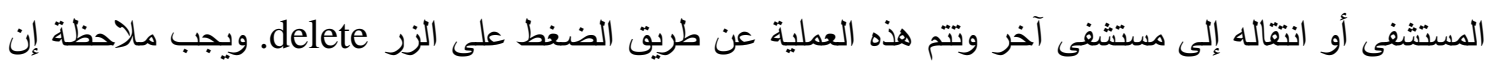
سجل الثخص الذي تعرض معلوماته في استمارة المعلومات هو السجل الذي يحذف من قاعدة البيانات .

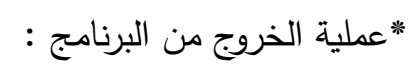

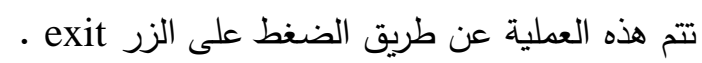

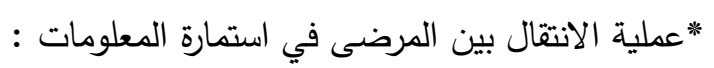
تتم هذه العملية عن طريق التعامل مع الأزرار الآتية: Next يتم الانتقال إلى السجل التالي في استمارة المعلومات : Prev . يتم الانتقال إلى السجل التالي في استمارة المعلومات : Last First : عملية التعرف على أعراض المريض :

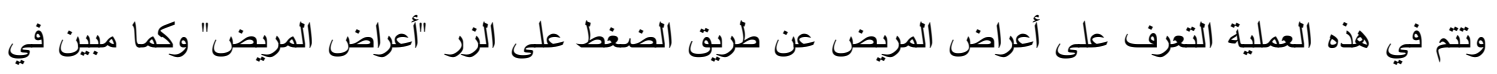
الواجهة الآتية (الثكل (8)) التي يظهر فيها اسم المريض وأعراض أعراض كما يلي:

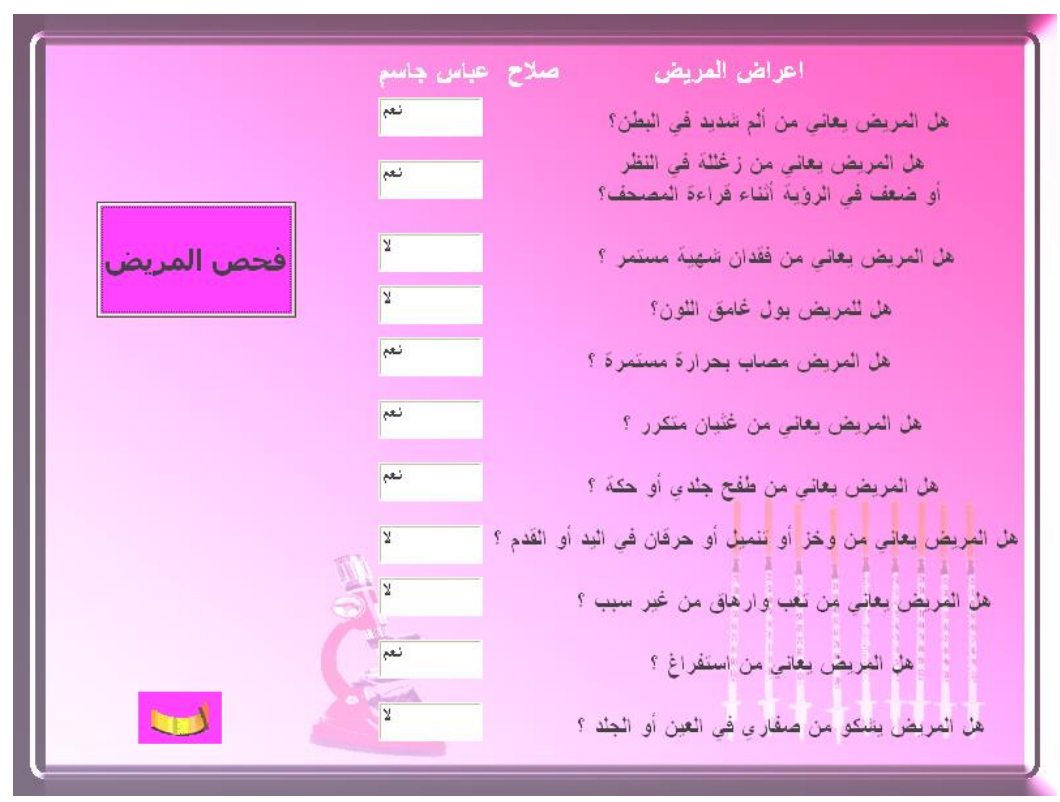

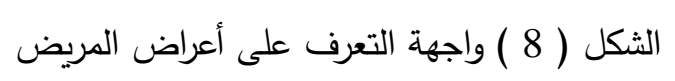

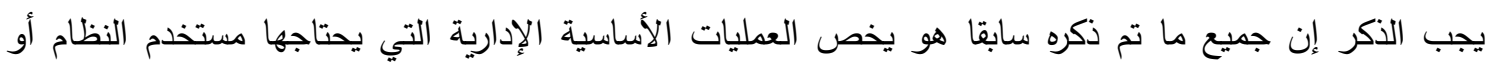

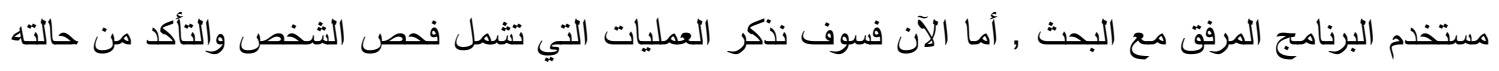

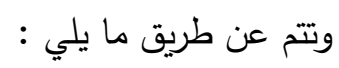




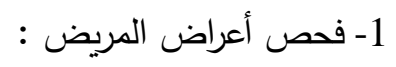

عند الضغط على الزر "فحص المريض " يتم تحويل أعراض المريض إلى (1-) إذا كان الجواب (لا) أو (1)

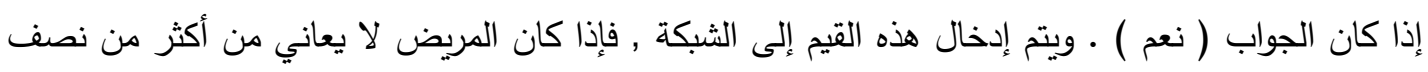

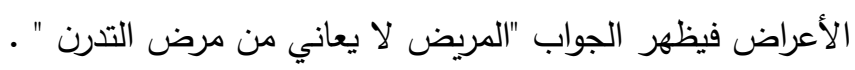

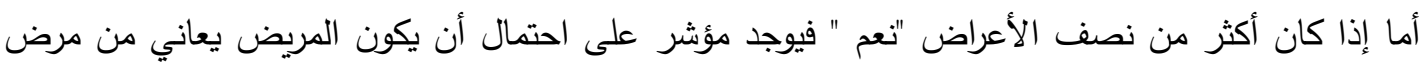

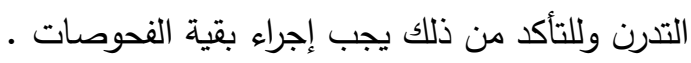

يعتبر إجراء فحص الدم هو عبارة عن فحص روتيني لمعرفة بعض المعلومات المهمة عن المريض وفصيلة

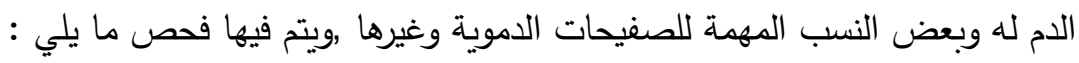
• Hb Pcv

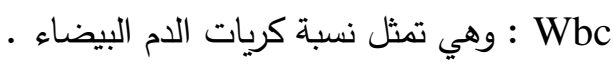
Esr • platelts

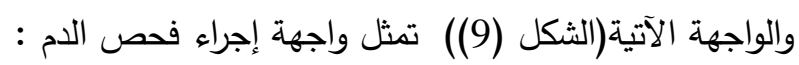

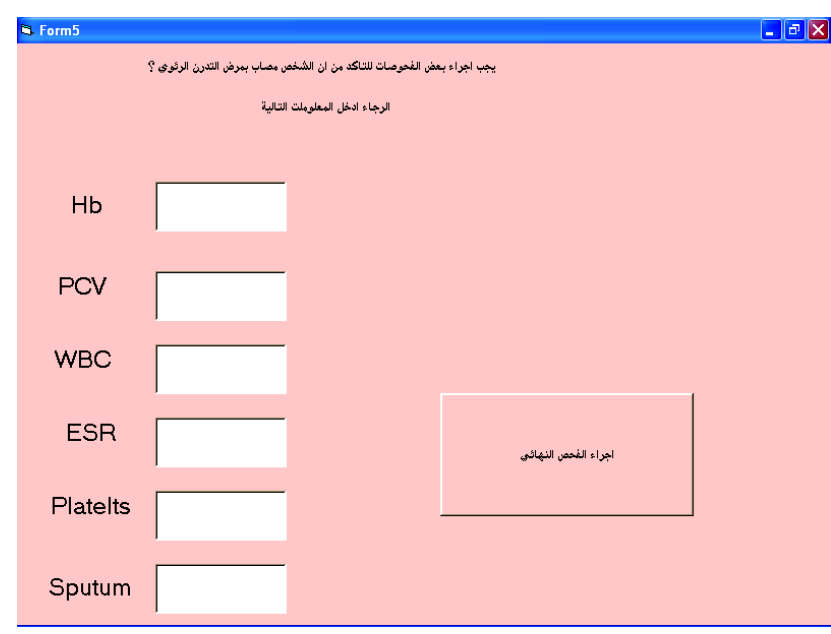

الشكل ( 9 ) واجهة فحص الدم

3- فحص القشع أو مخاط الرئتين (spatum) :

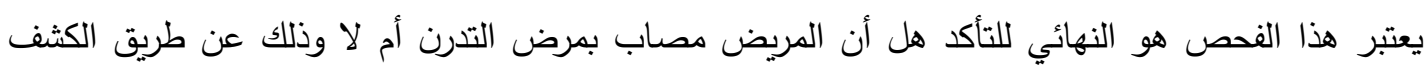
عن وجود عصيات تدرن (tubercle bacilli) في السائل المخاطي , فإذا كانت العصيات موجودة يعتبر المريض مصاب بمرض التدرن الرئوي • ويتم ذلك عن طريق الضغط على الزر "إجراء الفحص النهائي" . 
بعد الانتهاء من تصميم هذا النظام وتتفيذه على مجموعة من الأشخاص المصابين وغير المصابين فقد

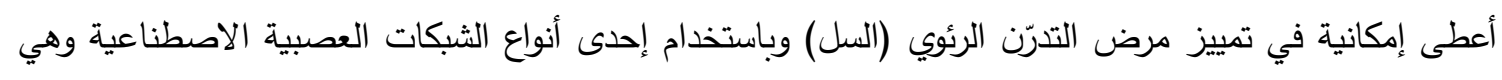

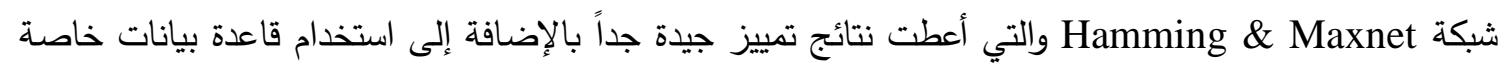
لخزن معلومات الأثخاص الذين يتم فحصهم وإمكانية استرجاعها في أي وقت عن طريق ربط قاعدة البيانات بالثبكة العصبية الاصطناعية. ولا بد من الإثارة إلى أنه يمكن تطبيق هذه الثبكة في تشخيص أمراض أخرى، وكذلك يمكن استخدام نوع آخر من الثبكات العصبية الاصطناعية في تشخيص مرض التدرّن الرئوي (السل). 
[1] Daniel G., 2007, Principle of Artificial Neural Network, World Scientific.

[2] Sarfraz M., 2005, Computer-Aided Intelligent Recognition Techniques and Applications, John Wiley \& Sons, Ltd.

[3] Krose B. \& Smagt P., 1996, An Introduction to Neural Networks.

الثبكات العصبية البينة الهندسية والخوارزميات - التطبيقات، ترجمة وإعداد علام زكي عيسى .

[5] Help of MATLAB 7.6, R, 2008 a.

$$
\text { Visual Basic }
$$

[7] http://www.sehha.com. 